\title{
The Construction of Seismic and Geological Studies' Cloud Platform Using Desktop Cloud Visualization Technology
}

\author{
Zhang Xiangyang ${ }^{*}$, Feng Chaomin and Wen Ling
}

PetroChina Research Institute of Petroleum Exploration \& Development-Northwest, Lanzhou, Gansu Province 730020 , P.R. China

\begin{abstract}
D visualization technology makes full use of 3D seismic information and properties. Using 3D graphics to describe all kinds of complex data fields and the data relation can be easily perceived, it is an important method of seismic and geological research. In petroleum exploration, the high performance graphics workstations are deployed to complete the interpretation work. The traditional application mode leads to the following problems: the utilization of resource is not balanced between different workstations; the lack of hardware, software and data resources sharing; and collaborative research is not convenient between experts in different places. By constructing seismic and geological studies' cloud platform using desktop cloud visualization technology, the traditional $\mathrm{C} / \mathrm{S}$ architecture software using mode change to $\mathrm{B} / \mathrm{S}$ mode, the platform improve the utilization rate of resources. The cloud platform realizes the seismic data interpretation work based on the $3 \mathrm{D}$ remote visualization technology, and the user needs only a desktop computer to complete all jobs. Through the cloud platform, users can access the software and hardware resources needed during the research by network, collaborative research of experts in different places will be very convenient, and the platform also provide science basis and data support for the comprehensive decisions.
\end{abstract}

Keywords: 3D visualization, collaborative research, desktop cloud visualization technology, cloud platform, software as a service.

\section{INTRODUCTION}

In 1970, Hilterman issued a paper "3D seismic simulation", and Welton put forward the concept of 3D seismic exploration, opening the era of the 3D technology in the field of seismic exploration. But due to the computer hardware conditions (such as small memory, slow speed limit), the application of this technology was restricted. After 1990 time metaphase, 3D visualization technology matured [1]. With the major software company Paradigm Geophysical, LandMark Graphics, Schlumberger/GeoQuest launched or upgrade the existing 3D visualization software, the 3D visualization technology has gradually become a new seismic interpretation technology [2]. 3D visualization technology makes full use of 3D seismic data and seismic attributes, using 3D graphics perceivable to describe all kinds of complex data field and the relationship of data .In 3D space, geological experts can be performed directly on the horizon of space domain automatic tracking, fault horizon analysis and spatial combination, sedimentary environment interpretation. The interactive interpretation seismic workstations are valuable tools for efficiently performing interpretation of large volumes of 3D seismic data. Visualization thus allows the geoscientist to think about large quantities of data, provides for speedy analysis [3], and the interpretation of 3D visualization technology has the advantages of short period, high accuracy $[4,5]$. Due to the nature of seismic data being noisy and visually complex, interpretations usually result in a multiple views of a feature. The workflow of seismic interpretation in the oil and gas industry usually involves collaborative visualization for experts to discuss and reconcile the different interpretations. Multiple teams (e.g. reservoir characterizations, modelling and reservoir management) often require access to interpretations [6]. This requires different experts gathered together, even the experts in different places together, or bring seismic project data to other places for collaborative research.

In the field of oil exploration, seismic data interpretation platform is often independent deployment. Through the allocation of local high performance workstations and high performance storage disk array realize the data storage and interactive display. This leads to duplication of investment, operation costs rise, low asset investment rate of return. On the other hand, due to the dispersion of seismic data and research results, and also seismic data volume is larger, so that data can not be achieved by experts in different locations during the real time collaborative research and scientific decision-making. Decentralized management of software and hardware resources results in the following issues: the utilization of resource is not balanced, the overall lack of resources; software and hardware resources can not be shared, repeated investment seriously; working convenience poor, unable to realize the collaborative research; results of data distribution is decentralized, no method to provide scientific decision support etc. 
In recent years, with the development of cloud computing technology, the software and hardware resources sharing is solved, the cloud computing provides the convenient method for decision support and collaborative research in the seismic data interpretation of different areas or places of experts. Using the Desktop Cloud Visualization technology, the traditional 2D/3D graphics display and seismic data interpretation work can be achieved through the network, realize the sharing of software and hardware resources, and users only need a simple desktop machine to complete all seismic data interpretation work, the user through the browser to get the software resources, storage resources needed in research, collaborative research work is also convenient in different places of experts, and provide methods for scientific decision.

\section{STUDY ON THE SOFTWARE AS A SERVICE TECHNOLOGY OF SEISMIC AND GEOLOGICAL STUDIES' CLOUD PLATFORM}

Cloud computing is a kind of soft, hardware resource usage pattern based on network. It is the integer-ration and development results of the load balance technology, virtualization technology, distributed computing, network computing and etc. The nature of cloud computing is the soft, hardware resource independent integration into a pool of resources, let the user through the network to realize the sharing and use of resources, it is the unified management, scheduling, and on-demand service to users.

Seismic and geological studies' cloud platform is a software as a Service (SAAS) private 3D cloud platform, the platform is divided into four layers: presentation layer, interface layer, application layer, the physical layer (Fig. 1).

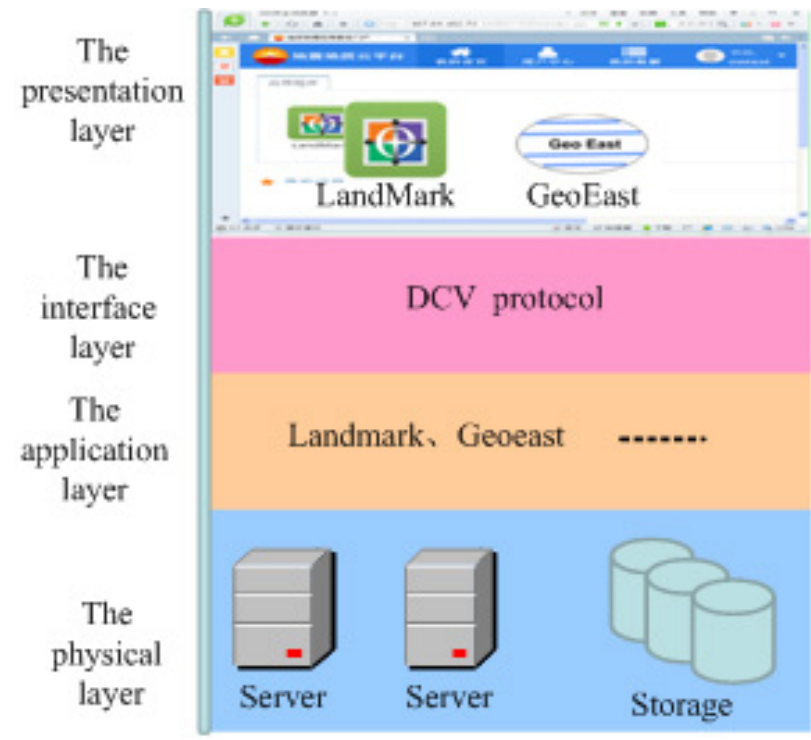

Fig. (1). This is the four level architecture diagram: presentation layer, interface layer, application layer, the physical layer.

1, the physical layer: realizing the unified deployment, centralized management of cluster server and storage systems, and through the load balancing technology, realize the load balance between the distributed cluster servers, improve the efficiency of software used;
2, the application layer: mainly refers to the installation of the application software and OS system, such as LandMark software, Geoeast software and etc. the platform adopts Linux system and VNC software to support multiuser and sessions sharing function.

3 , the interface layer: realizing the access interface of application software in the cloud platform, the platform adopts DCV protocol to realize the remote usage of $2 \mathrm{D} / 3$ Dgraphics software.

4, the presentation layer: through the WEB technology, software in cloud platform will be released to the internet, realizing the access of software based on the network. In the WEB portal, the user can see all of the available application software's' icons, and no longer need to work on a graphics workstation. So the cloud platform reduce the local hardware resources allocation and investment cost, at the same time, also makes the researcher get rid of the restrictions of space, scientific research personnel in any place can access the cloud platform, realize the online usage of software. Researchers can share their own session, at the same time, other researchers can easily access the session and see all software operation process through the network, so as to realize the collaborative study between experts in different locations.

\section{STUDY ON THE 3D REMOTE VISUALIZATION TECHNOLOGY OF SEISMIC AND GEOLOGICAL STUDIES' CLOUD PLATFORM}

Seismic and geological studies' cloud platform realizes remote 2D/3D graphic display and seismic interpretation using Desktop Cloud Visualization (DCV) technology. DCV technology is a kind of standard 2D/3D oriented interactive application protocol based on the network. Using DCV protocol can construct a 3D Cloud Application environment easily. The 3D cloud platform contains high performance graphics card, storage device, large memory, and fast I/O channel. The researchers only need to access the seismic and geological studies' cloud platform through the Web portal, and use the latest software. The traditional $\mathrm{C} / \mathrm{S}$ application model, software graphic information is processed by GPU, then display in the local screen after rendering. In the 3D cloud platform, Software graphic is processed and rendered in the data centre server, and sent to the remote screen display using the DCV protocol through the network. The 3D cloud platform realizes the transformation of the traditional application of $\mathrm{C} / \mathrm{S}$ mode to $\mathrm{B} / \mathrm{S}$ mode. The $\mathrm{DCV}$ protocol supports windows, Linux operating system, and also support GPU card sharing, greatly saves equipment investment, improve equipment utilization rate. DCV application framework is as follows (Fig. 2).

\section{THE CONSTRUCTION OF SEISMIC AND GEO- LOGICAL STUDIES' CLOUD PLATFORM}

Seismic and geological studies' cloud platform now has a successful application of LandMark and Geoeast software. It realizes the remote access of $2 \mathrm{D} / 3 \mathrm{D}$ software. Through the platform, researchers need not to install software locally such as Landmark, and carry out daily software maintenance, nor need to deploy high performance graphics workstations and local storage device. Data stored in the data centre, computing is also processed in the data centre, and researchers 


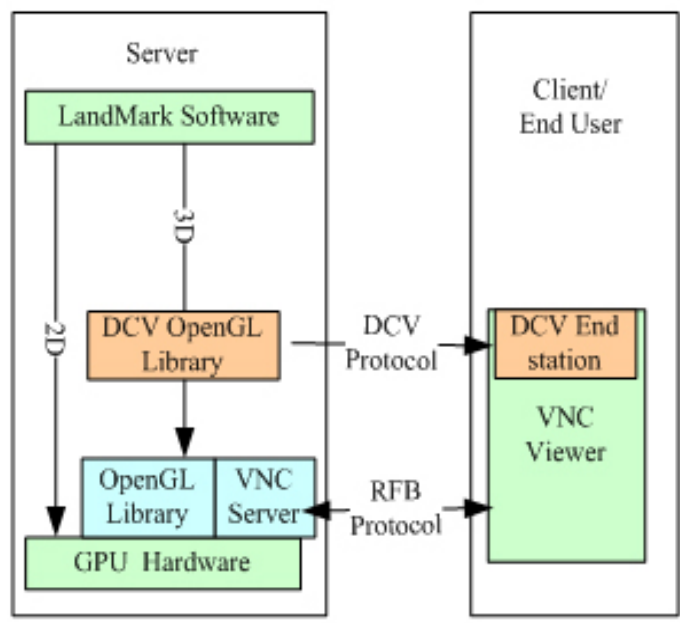

Fig. (2). DCV application framework: during the software using in the data centre, researchers connect to the Server host by VNC Viewer, the software's 3D graphic such as LandMark processed by DCV OpenGL Library, and send to the research's monitor by DCV protocol after rendering. So that researchers only need a compute to access the cloud platform to finish all interpretation works.

need only a PC which can connect to the Internet to complete seismic interpretation work. Researchers can use the software and data no matter when and where possible, regardless of software and data location, so as to improve the usage rate of LandMark software and hardware resource, avoid data synchronization and security problems during data migration. It greatly facilitates the researcher's daily interpretation work, provides a scientific method and data support for collaborative research and comprehensive deci- sion. This cloud platform can be conveniently to realize collaborative research for experts in different areas and places, providing intuitive, real-time, scientific research environment. In the cloud platform, project data can be used directly through the network, so as to provide scientific, real data to support decision making.

Seismic and geological studies' cloud platform realizes the sharing of software, hardware and data, changes the using way of software, solves the data sharing, inconsistency, and moving difficulty problems. In the cloud platform, all software and hardware resources are unified management and scheduling. Authorized users can use the software and hardware resources in the platform, which can increase the utilization of resources, avoid the consistency of data, reduce the data movement, provide a scientific decision-making method, and also greatly improve the efficiency of collaborative research (Figs. 3-6).

\section{CONCLUSION}

Construction of seismic and geological studies' cloud platform by using desktop cloud visualization technology, not only raise the utilization of the software and hardware resources, improve the degree of data sharing, solve the data inconsistency and movement difficult problems, but also can realize the remote usage of software, realize the remote interactive function of $2 \mathrm{D} / 3 \mathrm{D}$ seismic interpretation, greatly improve the convenience of seismic and geological research, researchers can access software and data whenever and wherever possible through the network, realize remote realtime collaborative study, provide methods and scientific support for the comprehensive decision.

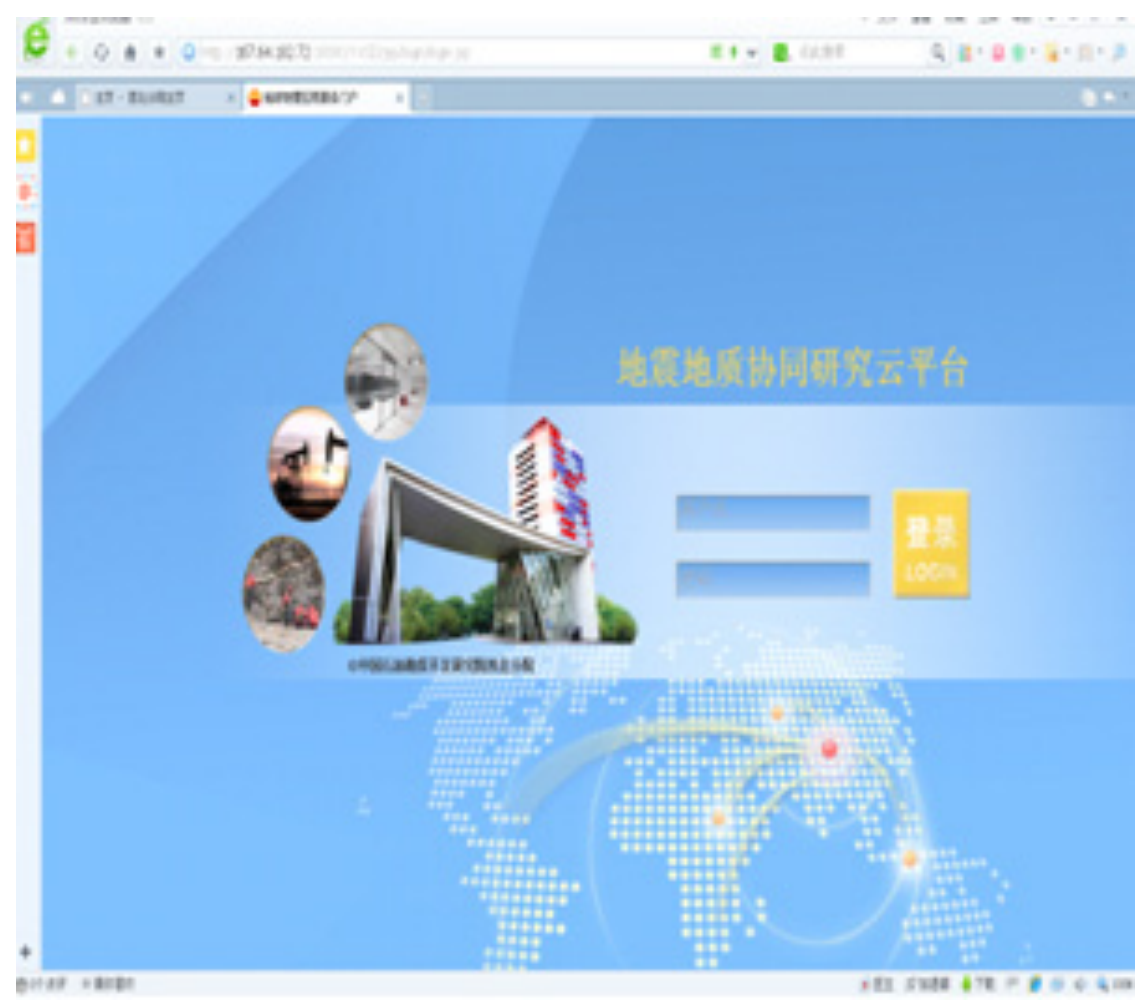

Fig. (3). Seismic and geological studies' cloud platform interface: The picture is the platform login interface, including the unified identity authentication and authorization verification of the user viewer. 


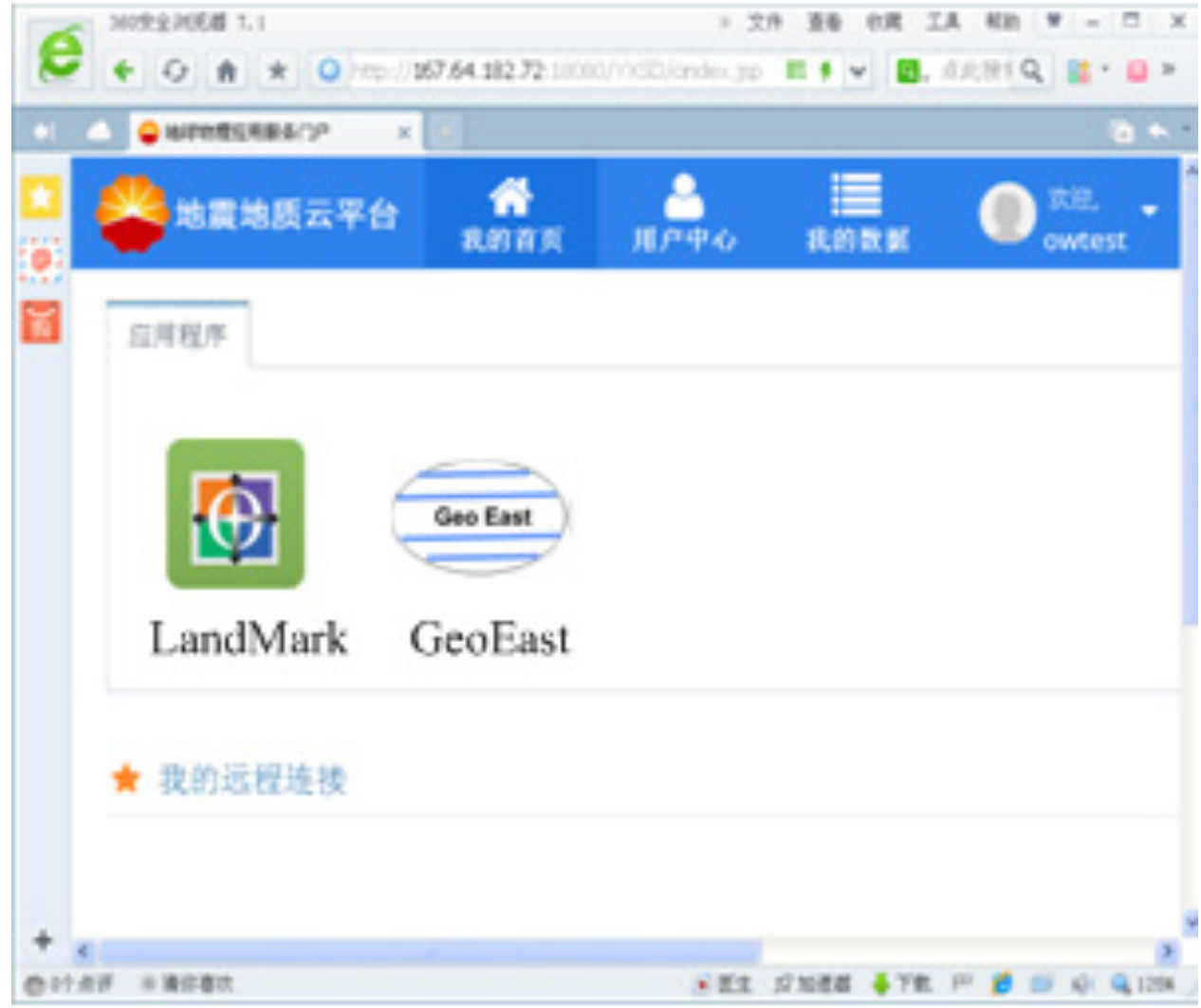

Fig. (4). Seismic and geological studies' cloud platform interface: the picture is the main user interface, the user can see all available software.

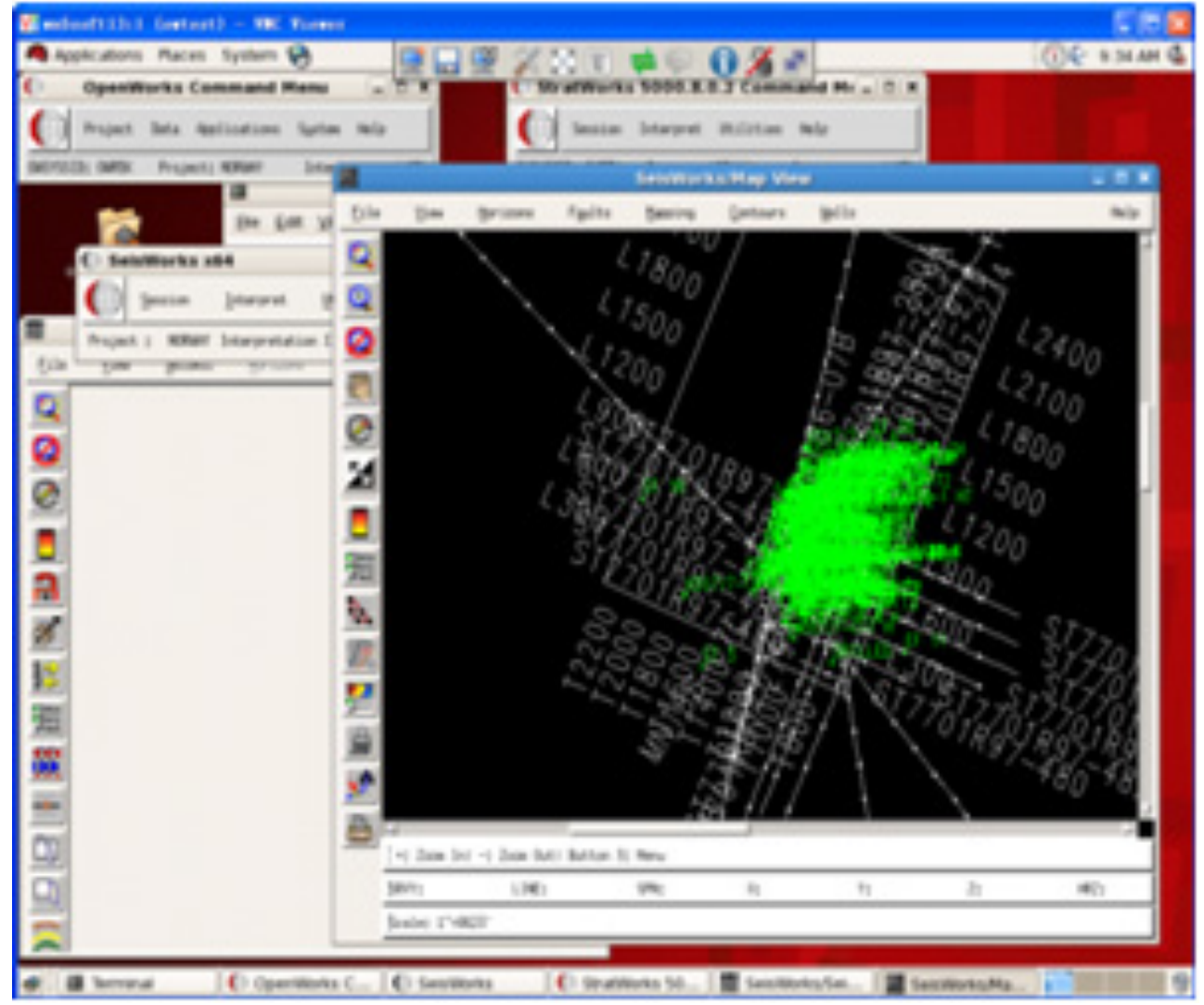

Fig. (5). Seismic and geological studies' cloud platform interface: \the following picture is the process of using LandMark 2D software in the remote software server by VNC viewer . 


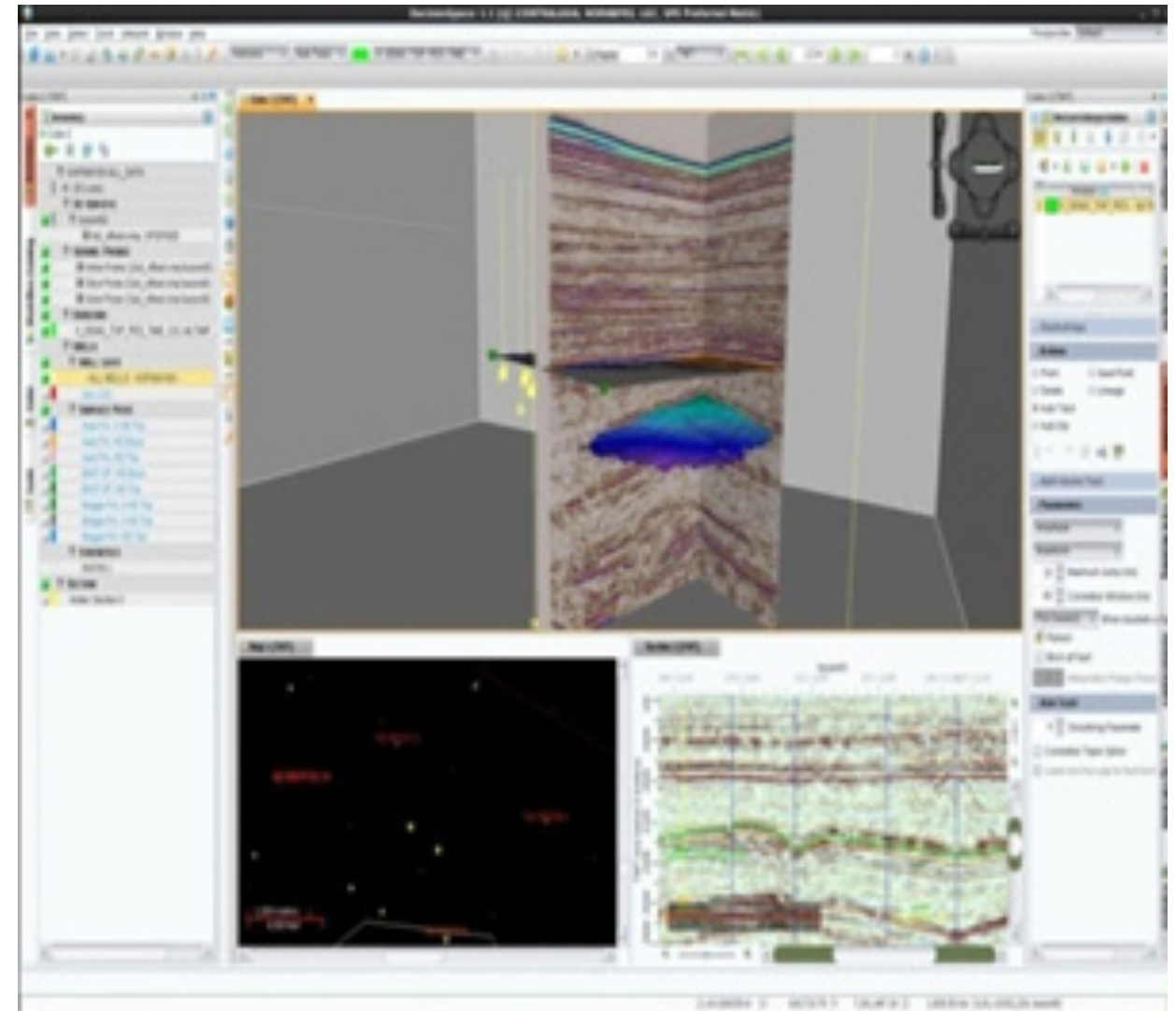

Fig. (6). Seismic and geological studies' cloud platform interface: \the following picture is the process of using LandMark 3D software in the remote software server by $\mathrm{VNC}$ viewer.

\section{CONFLICT OF INTEREST}

The authors confirm that this article content has no conflict of interest.

\section{ACKNOWLEDGEMENTS}

Declared none.

\section{REFERENCES}

[1] S. Jang, Z. Bo, "Application of 3-D visualization technique to seismic explanation," Journal of Ocean University Of China, vol. 34, no.1, pp. 147-152, 2004.
[2] R. W. Ward, "The future development trend of geophysical technology - American National Petroleum Council member view," The Leading Edge, vol. 15, no. 6, pp. 729-735, 1996.

[3] D. Gao, "3D seismic volume visualization and interpretation: An integrated workflow with case studies," Geophysics, vol. 74, no. 1, pp. 1-12, 2009.

[4] X. Wang, and H. Cao, "Elementary discussion on true 3D structural interpretation technique," Geophysical Prospecting For Petroleum, vol. 39 , no. 2 , pp. $89-94,2000$.

[5] G. D. Kidd, "Fundamentals of 3D seismic volume visualization,' "The Leading Edge," vol. 6, pp. 702-709, 1999.

[6] A. N. Aqeel, R. Masroor, I. Duncan, and B. John, "A data-centric approach to data provenance in seismic imaging data," In: $75^{\text {th }}$ EAGE Conference \& Exhibition incorporating SPE, Europec London, UK, 2013.

Received: June 10,2015

(C) Xiangyang et al.; Licensee Bentham Open.

This is an open access article licensed under the terms of the Creative Commons Attribution Non-Commercial License (http://creativecommons.org/licenses/by-nc/3.0/) which permits unrestricted, non-commercial use, distribution and reproduction in any medium, provided the work is properly cited. 